\title{
A search for rotational variations on trans-Neptunian objects ${ }^{\star}$
}

\author{
A. Alvarez-Candal, M. A. Barucci, F. Merlin, A. Guilbert, and C. de Bergh \\ LESIA, Observatoire de Paris, 92195 Meudon Cedex, France \\ e-mail: alvaro.alvarez@obsmp.fr
}

Received 20 June 2007 / Accepted 10 September 2007

ABSTRACT

\begin{abstract}
Aims. Our aim is to investigate the surface composition of TNOs. In particular we would like to confirm diverse spectral absorption features and to search for rotational inhomogeneities on one scattered disk object (26375) $1999 \mathrm{DE}_{9}$, two plutinos (38628) Huya and (47932) $2000 \mathrm{GN}_{171}$, and one centaur (83982) Crantor.

Methods. We observed the targets with a new instrument available at the VLT, the near-infrared integral field spectrograph SINFONI. We obtained complete near-infrared spectra between 1.4 and $2.4 \mu \mathrm{m}$ and compared them with data recorded previously by various groups.

Results. Two objects, (38628) Huya and (83982) Crantor, have evidence of an absorption feature in their spectra at $2.0 \mu \mathrm{m}$, probably associated with water ice. (83982) Crantor shows a feature at $2.3 \mu \mathrm{m}$ which could be associated with methanol. On the other hand, no features were found for (26375) $1999 \mathrm{DE}_{9}$ and (47932) $2000 \mathrm{GN}_{171}$ above the signal-to-noise ratio.

Possible rotational heterogeneity can be seen on two objects: (38628) Huya as the $2.0 \mu \mathrm{m}$ band is present on some spectra and absent on others, while (47932) $2000 \mathrm{GN}_{171}$ shows a flat reflectance in contrast to an absorption in the $\mathrm{H}$ region previously seen.
\end{abstract}

Key words. Kuiper Belt - techniques: spectroscopic

\section{Introduction}

The trans-Neptunian objects are commonly believed to be the most primitive objects of the Solar System and therefore carry clues about the physical and dynamical processes that acted since their formation.

Since the discovery of the first trans-Neptunian object (TNO) (Jewitt \& Luu 1993) about 1200 objects have been discovered, but only a relatively low number of them has been observationally studied. Photometry was the first technique used to study the surface properties of this population (see the recent review by Doressoundiram et al. 2007), followed by visible and nearinfrared spectroscopy (from 0.5 up to $2.4 \mu \mathrm{m}$ ). However, the database of spectroscopically well observed TNOs is still small. With the available observations, different spectral behaviors have been found from featureless spectra to objects that show signatures of ices, mainly $\mathrm{H}_{2} \mathrm{O}$ or $\mathrm{CH}_{4}$, (see, e.g., Barucci et al. 2007).

The diverse surface properties of the TNOs could be explained as a competition between different phenomena such as collisions, cometary activity, space weathering, and volatile loss due to atmospheric escape (Luu \& Jewitt 1996; Gil-Hutton 2002; Thébault \& Doressoundiram 2003; Schaller \& Brown 2007). Some tests of these models, especially the collisional evolution, are the study of objects with rotational inhomogeneities (e.g., Merlin et al. 2005; Licandro \& Pinilla-Alonso 2005), or the observation of targets likely to have suffered recent collisions (Alvarez-Candal et al. 2007).

According to their orbital characteristics the TNOs are commonly divided into sub-populations. In spite of more detailed definitions, we will only mention here the classical belt of TNOs, the resonant TNOs, captured in mean motion resonances with

\footnotetext{
* Based on observations collected at VLT Observatory Cerro Paranal of the European Southern Observatory, ESO in Chile, within the framework of program 077.C-0265.
}

Neptune, and the scattered disk objects (SDOs). In particular those resonant objects captured in the 3:2 mean motion resonance with Neptune are called plutinos, after (134340) Pluto.

Centaurs, on the other hand, are believed to be transition objects, probably dynamically and physically evolved TNOs which have found their way into the inner Solar System (e.g., Levison \& Duncan 1997). Thus, their surfaces resemble that of the TNOs, showing similar signatures and spectral behavior.

In what follows we will concentrate on four objects: the SDO (26375) $1999 \mathrm{DE}_{9}$, the plutinos (38628) Huya and (47932) 2000 $\mathrm{GN}_{171}$, and the centaur (83982) Crantor (formerly $2002 \mathrm{GO}_{9}$ ). It has been claimed that some features detected on their spectra can be explained by the presence of ices and/or hydrated minerals on the surface of these objects (Jewitt \& Luu 2001; de Bergh et al. 2004; Doressoundiram et al. 2005).

Jewitt \& Luu (2001) observed (26375) $1999 \mathrm{DE}_{9}$ in the nearinfrared. They found a spectrum with evidence of absorption features. Some features were associated with the presence of $\mathrm{Al}$ or $\mathrm{Mg}$ in minerals that incorporate $\mathrm{OH}$ within their structure, while a feature at $2.0 \mu \mathrm{m}$ was associated with water ice. Doressoundiram et al. (2003) suggested two possible models, one including a small amount of water ice and the other without. Unfortunately, they were only able to record an $\mathrm{H}$ spectrum. (38628) Huya has been widely observed. Its spectrum shows a generally featureless behavior in the infrared (Brown et al. 2000; Licandro et al. 2001), however water-ice has been included in the modeling of its spectrum (de Bergh et al. 2004). It was suggested, based on visible data, that this object shows rotational changes (de Bergh et al. 2004). In the same work the authors suggest that (47932) $2000 \mathrm{GN}_{171}$ might also show rotational variations. In a recent work, Brown et al. (2007) presented nearinfrared spectra of each of the three objects without any clear evidence of relevant features. 
Table 1. Observational circumstances.

\begin{tabular}{lccccccc}
\hline \hline Object & $V$ & Date & Exp. Time (s) & Airmass & Seeing (") & Solar Analog & Airmass \\
\hline (26375) 1999 DE & 20.3 & May 15, 2006 & 5400 & $1.132-1.328$ & $0.95-2.00$ & SA102 1081 & 1.110 \\
$(38628)$ Huya & 19.6 & Jul. 30, 2006 & 4800 & $1.158-1.746$ & $0.69-1.68$ & SA107 998 & 1.308 \\
$(47932)$ 2000 GN $_{171}$ & 20.6 & May 15, 2006 & 3000 & $1.023-1.040$ & $0.88-1.50$ & SA102 1081 & 1.110 \\
& 20.6 & May 28, 2006 & 4800 & $1.055-1.121$ & $0.88-2.00$ & SA107 998 & 1.106 \\
& 20.7 & May 31, 2006 & 3000 & $1.023-1.056$ & $0.76-1.28$ & SA107 998 & 1.114 \\
(83982) Crantor & 20.7 & May 15, 2006 & 3000 & $1.039-1.080$ & $0.84-1.21$ & SA102 1081 & 1.110 \\
\hline
\end{tabular}

Doressoundiram et al. (2005) observed (83982) Crantor. They obtained a spectrum with evidence of a band at $2 \mu \mathrm{m}$ associated with water ice. The authors included water ice in their model of the spectrum. Nevertheless, as the band of water ice at $1.5 \mu \mathrm{m}$ was not apparent, they suggested possible surface heterogeneity, due to the non-simultaneity of the observation of the different parts of the near-infrared spectrum. They also mentioned the possible existence of a band at $2.3 \mu \mathrm{m}$ that could be associated with methanol, but it was not included in the modeling of (83982) Crantor's spectrum because it led to a poor fit with the data.

In view of the particularities that the objects seem to share, we observed them using the ESO facility at the Paranal observatory with the near-infrared integral field spectrograph SINFONI. The observations, as well as the data reduction, are explained in the next section, while in Sect. 3 the spectra are discussed and compared to the data available in the literature. Finally, in the last section we present a short summary of the results.

\section{Observations and data reduction}

The data were taken during several observing runs between May and July of 2006 with the near-infrared integral field spectrograph SINFONI installed at the $8 \mathrm{~m}$ "Yepun" ESO-VLT facility at the Paranal Observatory in Chile. The instrument is equipped with an infrared detector of $2048 \times 2048$ pixels Hawaii 2 RG. In all cases the observations were performed using the $\mathrm{H}+\mathrm{K}$ grating, with a spectral resolution of about 1500 , and a plate scale of $0.25 \mathrm{arcsec} / \mathrm{spaxel}$ (field of view of $8^{\prime \prime} \times 8^{\prime \prime}$ ). The observations were carried on without using the adaptive optics mode, therefore being seeing limited.

The circumstances of observations of each target are reported in Table 1 . The reported $V$ magnitude was taken from the MPC ephemeris generator.

SINFONI slices the field of view into 32 image-slitlets which are re-directed toward the grating and then re-imaged on the detector. The reduction was performed using the SINFONI pipeline version $1.6^{1}$. The pipeline accounts for the whole correction procedures automatically: bad pixels maps, flat fielding, distortions, wavelength calibration, by using calibration data taken daily and also a basic set of static calibration and tables.

For all the science data, we perform the sky subtraction. If an independent sky image was taken during the observing block it is used as the reference sky, otherwise the sky of the science image having the closest time is used as reference sky. In the data presented here, the analog stars were observed along with independent sky exposures, while the object was not. It is important to stress that the pipeline includes routines that correct the contribution of the $\mathrm{OH}$ sky emission lines. Finally, the pipeline constructs the corresponding cubes of data by stacking the spectra in each slitlet in the right order and combines, by a

\footnotetext{
${ }^{1}$ http://www.eso.org/projects/dfs/dfs-shared/web/ sinfoni/sinfo-pipe-recipes.html
}

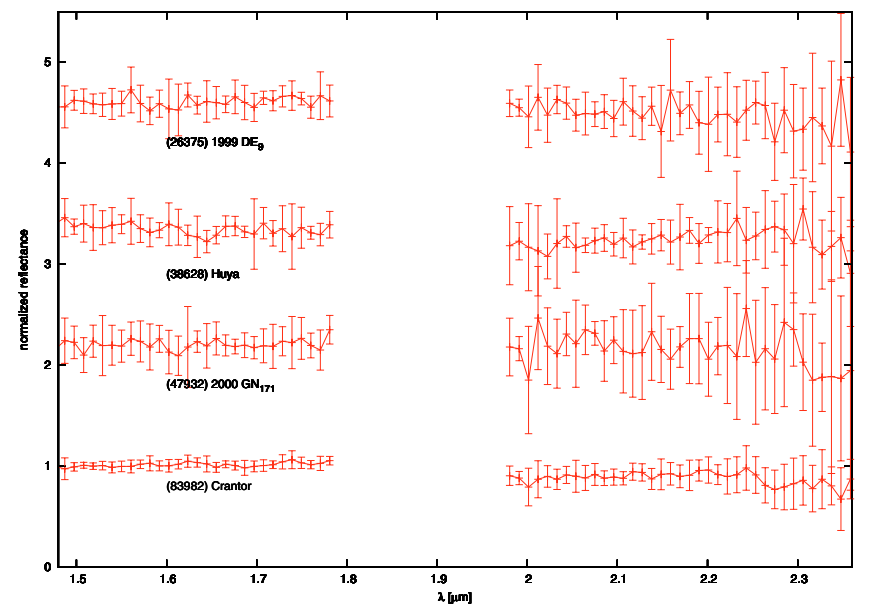

Fig. 1. Spectra of (26375) $1999 \mathrm{DE}_{9}$, (38628) Huya, (47932) 2000 $\mathrm{GN}_{171}$, and (83982) Crantor. All spectra were normalized to unity at $1.55 \mu \mathrm{m}$. An arbitrary offset was applied for clarity. The final resolution of the spectra after processing is about 200. The error bars show a one $\sigma$ deviation.

weighed mean, the dithered cubes to create the final science cube.

Once the science cube has been created, the spectrum is extracted using QFitsView, a tool developed at the Max Plank Institut für extraterrestrische Physik. The extraction is performed using an aperture selected as a compromise between the maximum gathered flux and the minimum residual sky contribution, in our case the size of the aperture was of about $1^{\prime \prime}$-radius for each of the objects. The spectra of the science objects were then divided by the spectra of the corresponding solar analog (see Table 1). They were cleaned using FORTRAN routines to eliminate bad pixels. This task is executed by measuring the median value and dispersion, flux $x_{\mathrm{m}}$ and $\sigma$, of the spectrum in a given range of wavelength of $\sim 0.025 \mu \mathrm{m}$ around $\lambda$. The criterion was:

$$
\text { if }\left\{\begin{array}{l}
\left|f \operatorname{lux}(\lambda)-\operatorname{flux}_{\mathrm{m}}\right|>2 \sigma \Rightarrow \operatorname{flux}(\lambda)=\text { flux }_{\mathrm{m}} \\
\left|\operatorname{flux}(\lambda)-\operatorname{flux}_{\mathrm{m}}\right|<2 \sigma \Rightarrow \operatorname{flux}(\lambda) \text { is kept. }
\end{array}\right.
$$

Finally, due to the high resolution of the spectra it is possible to degrade them by means of a re-binning increasing in this way the $\mathrm{S} / \mathrm{N}$ but at the cost of a lower spectral resolution. The re-binning was done over $0.0105 \mu \mathrm{m}$, accounting for a final spectral resolution of about 200. To each bin was associated the mean flux value and the standard deviation $\sigma$. These final data are shown in Fig. 1. The spectra were normalized to unity at $1.55 \mu \mathrm{m}$ and an arbitrary offset has been applied for clarity. The region between 1.79 and $1.98 \mu \mathrm{m}$ was eliminated due to strong telluric absorption.

In the following part of the paper we discuss each object individually. The obtained spectra are compared with those in the literature in order to analyze/compare the presence of features and/or check for possible rotational inhomogeneities. 


\section{Results}

Before starting the discussion on the different targets observed, it is important to stress that the different spectra shown below for comparison have been obtained with different instruments at different telescopes. In particular, one of the instruments, ISAAC, needs photometric calibrations in order to combine the $H$ and $K$ band spectra since they are taken separately, and this can affect the results, as will be discussed below. The other instruments record simultaneously the complete range from 1.4 up to $2.4 \mu \mathrm{m}$, and even a much wider range as for example NICS at the TNG.

\section{1. (26375) $1999 D E_{9}$}

This object has been observed by Jewitt \& Luu (2001) using NIRC at the Keck telescope. The authors claimed the presence of weak features in the near-infrared spectrum at 1.4, 1.6, 2.0, and $2.25 \mu \mathrm{m}$, some of them, especially at 1.4 and $2.25 \mu \mathrm{m}$, being assigned to the presence of either $\mathrm{Al}$ or $\mathrm{Mg}$ in minerals that incorporate $\mathrm{OH}$ within their structure.

In Fig. 2 we compare our spectrum (red continuous line) with that of Jewitt \& Luu (2001), blue dashed line, and that of Brown et al. (2007), black dotted-dashed line. We do not detect any obvious absorption feature in our spectrum, and in particular the ones proposed by Jewitt \& Luu. Unfortunately, the broad and shallow band at $2.0 \mu \mathrm{m}$ assigned to water ice in the Jewitt \& Luu's spectrum falls in the region that we discarded due to the strong atmospheric absorption. A visual comparison shows that our spectrum is flatter than the one from Jewitt \& Luu (2001) for wavelengths shorter than $1.8 \mu \mathrm{m}$. A decrease in the reflectance starting from $2.0 \mu \mathrm{m}$ is present in our spectrum and is not so evident in the spectrum of Jewitt \& Luu.

To quantify these differences we computed the spectral gradient $S^{\prime}$ as defined in Luu \& Jewitt (1996) both in the H and K spectral range independently. The values appear in Table 2. Also, in the last column we show the fractional change in the flux level of the spectra $D$, defined as one minus the ratio between the median reflectance in the range $1.7-1.8 \mu \mathrm{m}$ and the one in the range 2.0-2.1 $\mu \mathrm{m}$.

$S^{\prime}$ measures the change of a spectrum over a given wavelength range in units of percentage per $0.1 \mu \mathrm{m}$. Typical error in $S^{\prime}$ is about $1 \% /(0.1 \mu \mathrm{m})$. Note that, even if $S^{\prime}$ represents valuable quantitative information, it is strongly dependent on the wavelength interval considered. We used a common interval for all spectra: $1.50-1.75 \mu \mathrm{m}$ for $S_{\mathrm{H}}^{\prime}$ and $2.05-2.30 \mu \mathrm{m}$ for $S_{\mathrm{K}}^{\prime} . D$ gives information that can help to strengthen the existence of a water ice band, or not.

Note that the values of $S_{H}^{\prime}$ for the spectrum of (26375) 1999 $\mathrm{DE}_{9}$ of this work and Jewitt \& Luu are somewhat similar, the latest being the reddest. In $\mathrm{K}$ appear the highest differences, specially for the spectrum presented in this work, which is the bluest. We are not able to interpret the drop in K of our spectrum. The spectrum of Doressoundiram et al. (2003) was not included since they obtained only the $\mathrm{H}$ range. The spectrum of Brown et al. (2007) resembles that of Jewitt \& Luu.

The current known period of (26375) $1999 \mathrm{DE}_{9}$ is longer than $24 \mathrm{~h}$ (Sheppard \& Jewitt 2002), but with a great uncertainty. Therefore, it is not possible to compare the rotational phases of the recorded spectra to know if the non detection of features and the drop of reflectance at long wavelengths in our spectrum are due to rotational inhomogeneities.

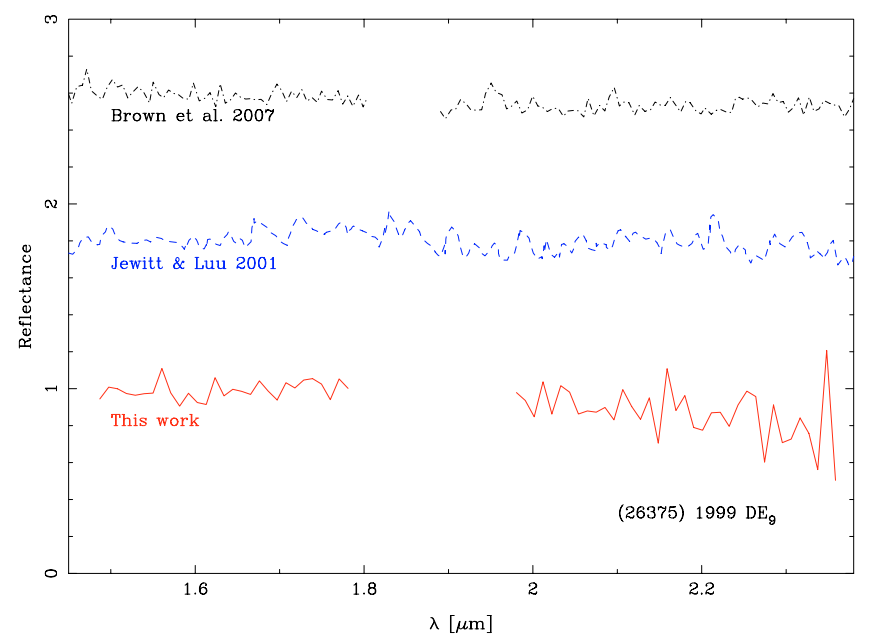

Fig. 2. Comparison between our spectrum of (26375) $1999 \mathrm{DE}_{9}$, red continuous line, that of Jewitt \& Luu (2001), blue dashed line, and that of Brown et al. (2007), black dotted-dashed line. All spectra were normalized to unity at $1.55 \mu \mathrm{m}$, an arbitrary offset of 0.8 has been applied to the flux scale for clarity.

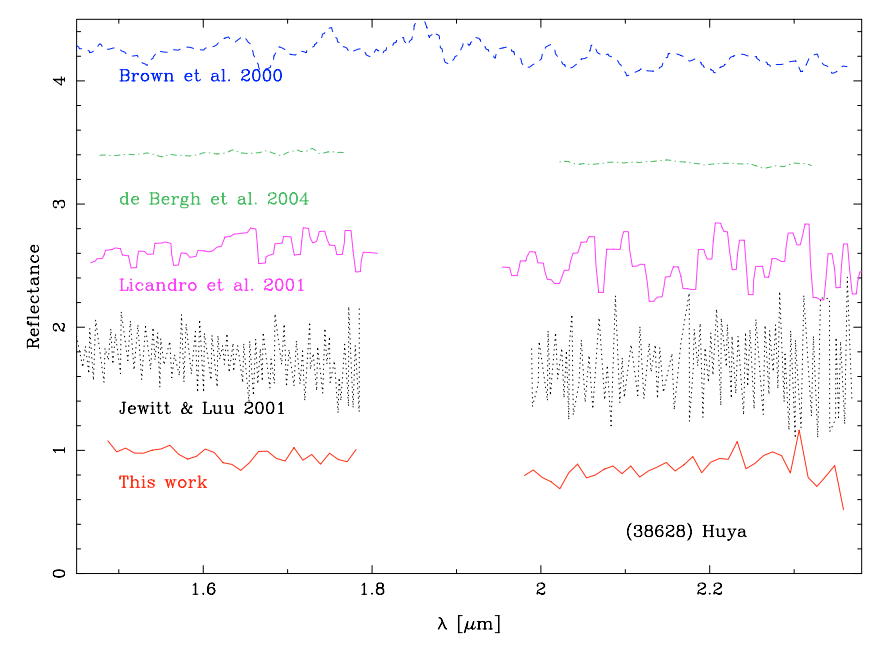

Fig. 3. Comparison between our spectrum of (38628) Huya (red continuous line) and that of Brown et al. (2000), blue dashed line, Jewitt \& Luu (2001), gray dotted line, Licandro et al. (2001), pink continuous line, and de Bergh et al. (2004), green dot-dashed line. All spectra were normalized to unity at $1.55 \mu \mathrm{m}$; an offset of 0.8 has been applied to the flux scale for clarity.

\section{2. (38628) Huya}

(38628) Huya is the best observed of the four targets since it is one of the brightest object in the trans-Neptunian region. In particular, several groups have observed it in the near-infrared (Brown et al. 2000 and 2007 using NIRC at the Keck telescope, Licandro et al. 2001 using NICS at the TNG, Jewitt \& Luu 2001 using CISCO at the Subaru, and de Bergh et al. 2004 using ISAAC at the VLT).

Differences found in visual spectra of (38628) Huya between 2001 and 2002 (Lazzarin et al. 2003 and de Bergh et al. 2004), and also differences in near-infrared spectra (e.g., Brown et al. 2000 and Jewitt \& Luu 2001) led to the suggestion that this object might show rotational heterogeneity. With this in mind we compare our near-infrared data with those of previous work in Fig. 3.

The main difference that appears while comparing the spectra is the presence or absence of an absorption band at about 
Table 2. Computed spectral gradients and $D$ parameter (see text).

\begin{tabular}{lcccc}
\hline \hline Object & Spectrum & $S_{\mathrm{H}}^{\prime}(\% / 0.1 \mu \mathrm{m})$ & $S_{\mathrm{K}}^{\prime}(\% / 0.1 \mu \mathrm{m})$ & $D(\%)$ \\
\hline (26375) 1999 DE D $_{9}$ & This work & 2.5 & -4.0 & 15 \\
& Jewitt \& Luu (2001) & 5.6 & -1.9 & 10 \\
& Brown et al. (2007) & -1.4 & 0.8 & 7 \\
\hline (38628) Huya & This work & -2.2 & 4.6 & 15 \\
& Brown et al. (2000) & 4.3 & -2.6 & 6 \\
& Jewitt \& Luu (2001) & -3.2 & 3.3 & 16 \\
& Licandro et al. (2001) & 14.1 & 3.5 & 14 \\
& de Bergh et al. (2004) & 1.5 & -1.4 & 9 \\
\hline (47932) 2000 GN (171 $_{1}$ & This work & 1.5 & 1.0 & -1 \\
& de Bergh et al. (2004) & 6.6 & 8.5 & -22 \\
& Brown et al. (2007) & 1.6 & 2.1 & -8 \\
\hline (83982) Crantor & This work & 1.2 & -2.8 & 14 \\
& Doressoundiram et al. (2005) & 8.3 & -8.1 & 13 \\
\hline
\end{tabular}

$2.0 \mu \mathrm{m}$, possibly associated with water ice. Neither Brown et al. (2000) nor Jewitt \& Luu (2001) suggested the existence of that band in their spectra. On the other hand Licandro et al. (2001) mentioned it but did not associate it with water ice. Finally, de Bergh et al. (2004) fitted the spectrum of Huya with a model that includes water ice. The spectrum presented in this paper shows an absorption feature at $2.0 \mu \mathrm{m}$.

From a quantitative point of view it is possible to see that the spectra of Jewitt \& Luu, Licandro et al., and that presented in this work have similar values of $D$. A closer inspection shows that Jewitt \& Luu's spectrum and the one presented in this work are in fact very similar, as indicated by the spectral gradients in Table 2 .

Therefore, the only spectrum that apparently does not have the absorption feature at $2.0 \mu \mathrm{m}$ is that of Brown et al. (2000). (38628) Huya has a poor determination of its rotational properties (Sheppard \& Jewitt 2002). It would be interesting to have a good determination of the rotational period of (38628) Huya in order to fit the different observations in rotational phase and determine whether or not geographically different regions of this body have been observed.

\section{3. (47932) $2000 \mathrm{GN}_{171}$}

Table 1 shows that the conditions under which (47932) 2000 $\mathrm{GN}_{171}$ was observed were not ideal with seeing ranging up to $2^{\prime \prime}$. This, combined with the faintness of the object, makes hard to believe in the reality of the feature that appears in our spectrum starting at $2.3 \mu \mathrm{m}$.

(47932) $2000 \mathrm{GN}_{171}$ was observed by de Bergh et al. (2004) in the near-infrared. Figure 4 shows a comparison between their near-infrared spectrum and the one obtained in the present work. There exist differences in the spectral shapes of these spectra. While in the de Bergh et al.'s spectrum there apparently exists evidence of an absorption feature (important difference between the $H$ and $K$ band flux levels; $D \sim-20 \%$ ), our spectrum is flat ( $D \sim-1 \%$ ). Recent published observations (Brown et al. 2007) show a flat continuum in agreement with our observations $(D \sim$ $-8 \%$ ). This last spectrum is also presented in Fig. 4.

It should be noted however that de Bergh et al. $H$ and $K$ spectra were recorded separately, using ISAAC at the VLT, on two different nights and they were combined together by photometric calibrations made on only one night due to non-photometric conditions on the other night. These photometric measurements are used to calibrate the reflectance levels of the individual spectra.

On this object has been claimed the presence of rotational variations based on the appearance and subsequent

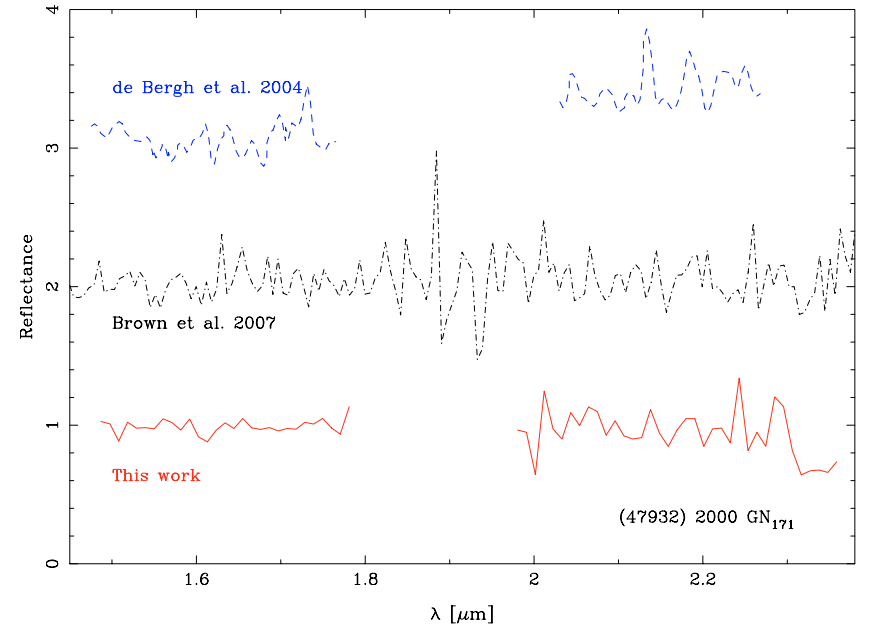

Fig. 4. Comparison between our spectrum of (47932) $2000 \mathrm{GN}_{171}$, red continuous line, and those of Brown et al. (2007), black dot-dashed line, and de Bergh et al. (2004), blue dashed line. All spectra were normalized at $1.55 \mu \mathrm{m}$, an offset of 1.0 has been applied to the flux scale for clarity.

disappearance of features in the visible (see Lazzarin et al. 2003 and de Bergh et al. 2004). Fornasier et al. (2004) searched for these rotational inhomogeneities. They covered about $60 \%$ of the rotational period ([8.329 \pm 0.005$]$ h, Sheppard \& Jewitt 2002), showing a quite homogeneous behavior. The only spectrum that looks different is that of Lazzarin et al. (2003) whose estimated rotational phase falls outside the range spanned by Fornasier et al.'s observations. New spectra in the visible for this object are currently under analysis.

Unfortunately, is not possible to compare the rotational phases of the de Bergh et al.'s observations and the ones presented in this work due to the long time interval between them. Simultaneous lightcurve and rotational spectral observations should be done in order to confirm or refute the rotational heterogeneity.

\section{4. (83982) Crantor}

This object had been observed previously in the near-infrared by Doressoundiram et al. (2005). The authors used ISAAC at the VLT which, as discussed above, needs photometric calibrations in order to combine the $\mathrm{H}$ and $\mathrm{K}$ spectra. The spectrum shows signatures, specially at $2.0 \mu \mathrm{m}$ modeled with water ice. They also suggest a band at $2.3 \mu \mathrm{m}$, associated with methanol. The 


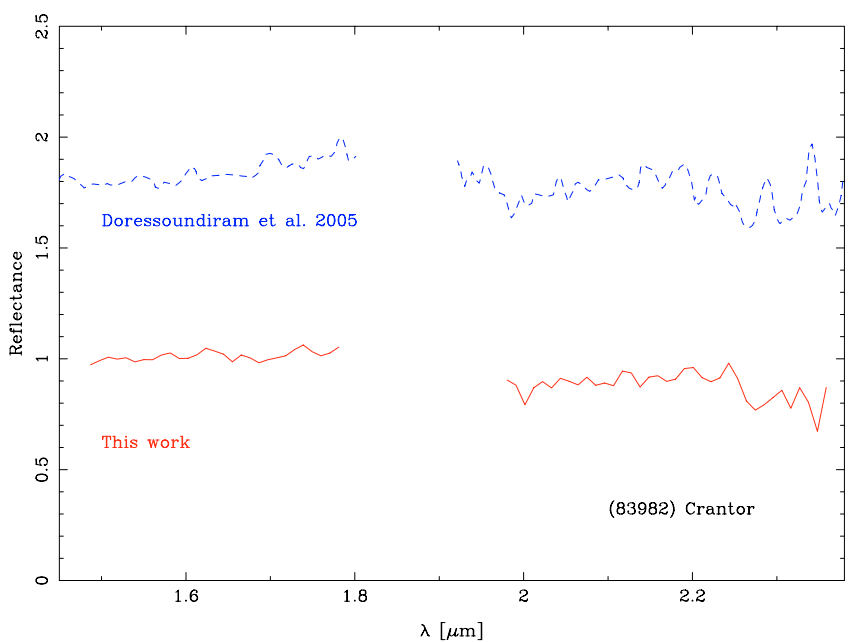

Fig. 5. Comparison between the spectrum of (83982) Crantor, red continuous line and that of Doressoundiram et al. (2005), blue dashed line. All spectra were normalized at $1.55 \mu \mathrm{m}$, an offset of 0.8 has been applied in the flux scale for clarity.

authors did not find a model that satisfactorily fits the structures seen in their spectrum. A model including water ice fails to reproduce the $\mathrm{H}$ part of the spectrum and the structure at $2.3 \mu \mathrm{m}$. Including methanol in the model does not improve the quality of the fit. They suggested that, due to the non-simultaneity of their recorded data (see Tables 2 and 3 in Doressoundiram et al. 2005), one possible reason for this apparent incompatibility is the observations of different parts of the surface of (83982) Crantor.

We compare their spectrum with ours in Fig. 5. As they did, we also detect the presence of an absorption feature at $2.0 \mu \mathrm{m}$, and no signature at $1.5 \mu \mathrm{m}$. In addition there is more clear evidence of an absorption structure at $2.3 \mu \mathrm{m}$ in our spectrum. There is an important difference in $\mathrm{H}$, as seen in Table 2, the spectrum of Doressoundiram et al. being the reddest. Both spectra have similar values of $D$, about $15 \%$.

We compare the spectrum of (83982) Crantor with the spectra of crystalline water ice and methanol (Fig. 6). Note that the band of methanol at $2.3 \mu \mathrm{m}$ fits with the position of the band seen in our spectrum. The $2.0 \mu \mathrm{m}$ band of water ice also fits with the position of the decrease in the flux level of Crantor's spectrum. Regarding the water ice band at $1.5 \mu \mathrm{m}$, note that this band is fainter than that at $2.0 \mu \mathrm{m}$ and may possibly be hidden in the noise of our spectrum.

The rotation period of (83982) Crantor is $13.14 \mathrm{~h}$ (Ortiz et al. 2003), but with a very large uncertainty. Therefore, although Doressoundiram et al.'s spectrum and the one presented in this work look similar, rotational inhomogeneities cannot be discarded.

\section{Conclusions}

We obtained near-infrared spectra of two plutinos, one SDO and one centaur with the SINFONI integral field spectrograph. A brief resume of the main results and comparisons with previous data from the literature is given below.

1. (26375) $1999 \mathrm{DE}_{9}$ : the spectrum presented in this paper is featureless within its noise. The only relevant structure that appears is a decrease in the reflectance starting from $2.0 \mu \mathrm{m}$. We do not confirm the detection of the features proposed by Jewitt \& Luu (2001), but it is not possible to rule them out either, thus their existence remains an open question.

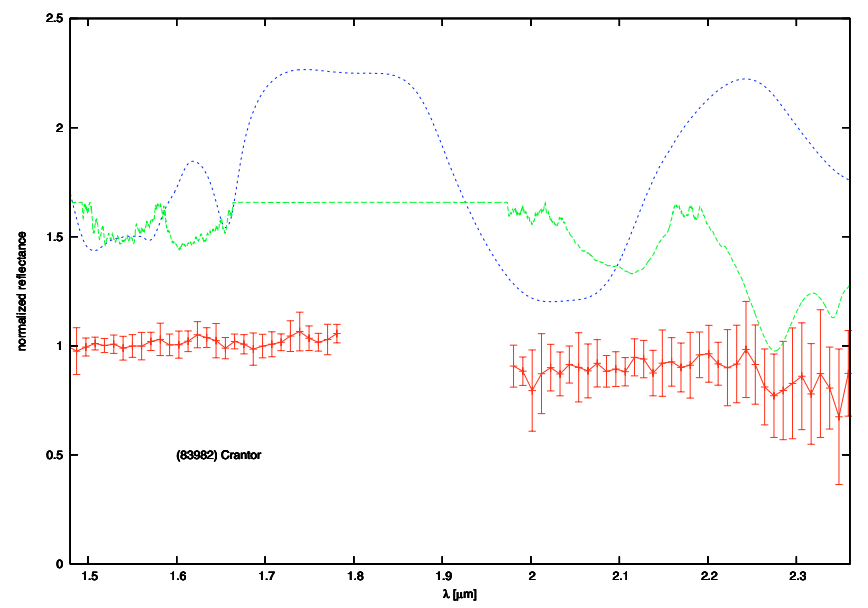

Fig. 6. Comparison of the spectrum of (83982) Crantor, red continuous line, with those of crystalline water ice, blue dashed line, and methanol, light-blue dot-dashed line. Both ices' spectra were obtained for a grain size of $10 \mu \mathrm{m}$ and a temperature of $40 \mathrm{~K}$ (B.Schmitt personal communication, Grundy \& Schmitt 1998).

2. (38628) Huya: there exists evidence of an absorption band at $2.0 \mu \mathrm{m}$, probably a shallow water ice band. The spectrum also shows a sharp drop in the reflectance starting from $2.3 \mu \mathrm{m}$, but this is probably due to noise.

Apparently, there are variations in the presence of the $2.0 \mu \mathrm{m}$ band. A better determination of its rotational properties is needed in order to make sure that it is due to heterogeneity.

3. (47932) $2000 \mathrm{GN}_{171}$ : the combined spectrum has a flat shape without apparent absorption bands. Regarding the structure that appears in the end of the $\mathrm{K}$ region, this is probably due to high noise rather than a real band.

The comparison with de Bergh et al. (2004) in the nearinfrared, shows that rotational inhomogeneities cannot be ruled out. In this spectral range the rotational period has not been totally covered, therefore it is possible that the data presented here correspond to different parts of the surface that those of de Bergh et al.

4. (83982) Crantor: there is evidence of absorption features at 2.0 and $2.3 \mu \mathrm{m}$. The first band is associated with water ice, while the second might be associated with methanol. This behavior was already seen by Doressoundiram et al. (2005). We do not have enough data to search for rotational heterogeneity.

In comparing the different spectra of the four objects there can be seen the performance of different telescopes and instruments. As stated in Sect. 2 SINFONI@VLT allows one to obtain at once a complete spectrum from 1.4 to $2.4 \mu \mathrm{m}$ with a relatively high $\mathrm{S} / \mathrm{N}$ for objects with $\mathrm{V}$ magnitude up to 20-21. The high initial spectral resolution, 1500, allows one to perform a smoothing in order to increase $\mathrm{S} / \mathrm{N}$, while keeping high enough resolution to obtain valuable information. The importance of obtaining good spectral coverage simultaneously without relying on further calibrations was discussed while comparing with ISAAC, also at the VLT.

As mentioned in Sect. 2 of this paper, the observations were carried out in the non adaptive optics mode, i.e., they are seeing limited. For future observations the use of laser guide stars, allowing the utilization of the adaptive optics mode, should improve the quality of observations. SINFONI, as well as the other instruments, are becoming fundamental tools for the study and understanding of the icy bodies of the outer Solar System. 
Acknowledgements. We would like to thank $\mathrm{K}$. Barkume and $\mathrm{M}$. Brown for kindly providing the spectrum of (47932) $2000 \mathrm{GN}_{171}$. A.A.C. would like to acknowledge financial support by ESA through a trainee-ship at the LESIA/Observatoire de Paris. We would also like to acknowledge J. Licandro for his constructive comments.

\section{References}

Alvarez-Candal, A., Jones, D., Lazzaro, D., Williams, I. P., \& Melita, M. 2007, A\&A, 466, 749

Barucci, M. A., Brown, M. E., Emery, J. P., \& Merlin, F. 2007, Composition and surface properties of TNOs and Centaurus, in The Solar System beyond Neptune, ed. A. Barucci, H. Boehnhardt, D. Cruikshank, \& A. Morbidelli (Tucson: Univ. of Arizona Press), in press

Brown, M. E., Blake, G. A., \& Kessler, J. E. 2000, ApJ, 543, L163

Brown, M. E., Barkume, K. M., Ragozzine, D., \& Schaller, E. L. 2007, Nature, 446, supplementary information

de Bergh, C., Boehnhardt, H., Barucci, M. A., et al. 2004, A\&A, 416, 791

Doressoundiram, A., Tozzi, G. P., Barucci, M. A., et al. 2003, AJ, 125, 2721

Doressoundiram, A., Barucci, M. A., Tozzi, G. P., et al. 2005, Plan. Space Sci., 53,1501
Doressoundiram, A., Boehnhardt, H., Tegler, S. C., \& Trujillo, C. 2007, Color properties and trends of the trans-Neptunian objects, in the Solar System Neptune, ed. A. Barucci, H. Boehnhardt, D. Cruikshank, \& A. Morbidelli (Tucson: Univ. of Arizona Press), in press

Fornasier, S., Doressoundiram, A., Tozzi, G. P., et al. 2004, A\&A, 421, 353

Gil-Hutton, R. 2002, Plan. Space. Sci., 50, 57

Grundy, W. M., \& Schmitt, B. 1998, JGR 103, E11, 25809

Jewitt, D. C., \& Luu, J. X. 1993, Nature, 362, 730

Jewitt, D. C., \& Luu, J. X. 2001, AJ, 122, 2099

Lazzarin, M., Barucci, M. A., Boehnhardt, H., et al. 2003, AJ, 125, 1554

Levison, H. F., \& Duncan, M. J. 1997, Icarus, 127, 13

Licandro, J., \& Pinilla-Alonso, N. 2005, AJ, 630, L93

Licandro, J., Oliva, E., \& Di Martino, M. 2001, 373, L29

Luu, J., \& Jewitt, D. 1996, AJ, 112, 2310

Merlin, F., Barucci, M. A., Dotto, E., de Bergh, C., \& Lo Curto, G. 2005, A\&A, 444, 977

Ortiz, J. L., Gutiérrez, P. J., Casanova, V., \& Sota, A. 2003, A\&A, 407, 1149

Schaller, E. L., \& Brown, M. E. 2007, ApJ, 659, L61

Sheppard, S. S., \& Jewitt, D. C. 2002, AJ, 124, 1757

Thébault, P., \& Doressoundiram, A. 2003, Icarus, 162, 27 